\title{
Amifostine ameliorates cerebral ischaemia-reperfusion injury via p38-mediated oxidative stress and mitochondrial dysfunction
}

\author{
Huifeng Cheng, Miaojun Lv, Rulin Mi, Guofang Xue
}

Second Hospital of Shanxi Medical University, China

\begin{abstract}
Amifostine is a cytoprotective compound that is beneficial in ischaemic stroke cases. However, the neuroprotective effect of amifostine on ischaemia/reperfusion (I/R)-induced brain injury and its underlying mechanism are still poorly understood. Herein, we constructed an animal model of middle cerebral artery occlusion and reperfusion (MCAO/R) injury and an in vitro model of oxygen and glucose deprivation and reperfusion (OGD/R) injury. After administration of amifostine, we found significant improvements in neurological deficits, infarct size, and cerebral oedema. Moreover, amifostine alleviated histopathological alteration and increased the number of surviving neurons. Biochemical analysis showed that treatment with amifostine obviously improved the brain damage of MCAO/R mice, as manifested by a decrease in reactive oxygen species (ROS) and malondialdehyde (MDA) generation, and an increase in superoxide dismutase (SOD) activity. Moreover, amifostine decreased the mitochondrial membrane potential $(\triangle \Psi \mathrm{m})$ loss, and cytochrome c escaping to cytoplasm, but increased the ATP level. In vitro, amifostine also showed an antioxidant effect, which was reflected by the reduced ROS generation, decreased mitochondrial superoxide generation, increased total SOD, SOD 1 (CU/Zn SOD, cytoplasmic SOD), and SOD2 (mitochondrial SOD) activities, and decreased $\Delta \Psi m$ loss. Furthermore, amifostine suppressed neuronal apoptosis, accompanied by the reduction of Bax, cleaved caspase-9, cleaved caspase-3, and Bcl-2 upregulation. Amifostine also reduced the expression of p-p38 (Thr 180/Tyr 182) in vivo and in vitro. In short, amifostine exhibits a protective effect on cerebral I/R damage through modulating p38-related oxidative stress, mitochondrial dysfunction, and apoptosis.
\end{abstract}

Key words: amifostine, ischaemia-reperfusion injury, brain, mitochondrial dysfunction, neuron.

\section{Introduction}

Cerebral ischaemia is the third major cause of death in the world, following cardiovascular diseases and tumours, in terms of morbidity and mortality [22,32]. At present, early blood supply or reperfusion is generally considered to be the most effective treatment for cerebral ischaemia in the clinic. However, reperfusion following ischaemia may result in severe brain failure [9]. In the past few decades, investigators have discovered that the underlying mechanisms of ischaemia-reperfusion (I/R) injury are closely connected with the generation of oxygen-free radicals, subsequent oxidative stress response, inflammatory damage, and eventually apoptosis $[15,19,22]$. In detail, ischaemia injury is caused by pathological conditions, including the production of a large amount of 
reactive oxygen species (ROS) and the activation of metabolic enzymes, respiratory chain, and adenosine triphosphate (ATP) synthase, eventually leading to cerebral infarct, neurological deficit, and neuronal death [10]. Therefore, it is imperative to find effective drugs to reduce the process of cerebral I/R injury.

Amifostine, formerly known as WR-2721, chemically known as ethanethiol, 2-[(3-aminopropyl) amino]-dihydrogen phosphate, is an inorganic thiophosphate with the potential of being a cytoprotectant and therapeutic agent [30]. It can scavenge free radicals and donate hydrogen to protect against radiation in animals and cells [28]. Increasing studies have shown that amifostine reduces the neurotoxicity induced by paclitaxel [35], cisplatin [40], cyclophosphamide [21], and platinum [27] in vitro. Moreover, amifostine alleviates hippocampal neurogenesis and brain recognition memory impairment [18]. More importantly, amifostine has been found to ameliorate ischaemic injury. One previous study described preconditioning with amifostine in prevention of heart I/R damage, depending on its antioxidant property [39]. Similarly, the protective effect of amifostine is also found in kidney [20] and spinal cord [7] with I/R injury. Additionally, amifostine has an anti-apoptotic effect, which is related to its regulation in apoptosis-related proteins [39].

In our study, we focused mainly on whether amifostine has antioxidant and anti-apoptotic effects on brain I/R injury, which might provide a candidate drug for minimising I/R injury.

\section{Material and methods \\ Animal model}

Male C57BL/6 mice weighing approximately $24 \mathrm{~g}$ (10 12 weeks old) were acquired from Liaoning Changsheng Biotechnology Co., Ltd (Liaoning, China). They were kept at the room temperature $\left(25 \pm 1^{\circ} \mathrm{C}\right)$ and humidity of $45-55 \%$. Before the operation, the mice were free to receive food and water, and were fully adapted to their surroundings. All animal experiments are approved by the Animal Care Committee of the Second Hospital of Shanxi Medical University.

The establishment of the MCAO model in mice was based on the description in previous studies $[5,13]$. After ischaemia, the nylon thread in the external carotid artery was carefully drawn out for reperfusion. The control animals were also given the above surgical procedure without carotid ligation. For amifostine administration, the experimental animals were intraperitoneally injected with amifostine at a dose of $200 \mathrm{mg} / \mathrm{kg}$ or $400 \mathrm{mg} / \mathrm{kg}[2,17]$ (HYB0639, MedChemExpress [MCE], New Jersey, USA) $30 \mathrm{~min}$ before ischaemia operation. After $1 \mathrm{~h}$ of ischaemia, the reperfusion was administrated for $24 \mathrm{~h}$. Then, the brains of each group of mice were collected and evaluated.

\section{Oxygen and glucose deprivation/ reoxygenation}

Oxygen and glucose deprivation/reoxygenation (OGD/R) in primary hippocampal neurons was achieved according to the previous method [43]. The hippocampal tissues were minced, trypsinised, and then kept in DMEM (12100-46, Gibco, New York, USA) containing additional $10 \%$ foetal bovine serum (FBS; 04-001-1ACS, BI, Israel). After centrifugation for $5 \mathrm{~min}$, the neurons were mixed and maintained in DMEM supplemented with 2\% B27 (17504044, ThermoFisher, Waltham, USA) and $0.5 \mathrm{mM}$ glutamine (HY-B0639, MCE) until the final cell density was $90 \%$.

After pretreatment with $50 \mu \mathrm{M}$ or $100 \mu \mathrm{M}$ amifostine [39], the primary hippocampal neurons were cultured in glucose-free DMEM $\left(95 \% \mathrm{~N}_{2} / 5 \% \mathrm{CO}_{2}\right.$, $37^{\circ} \mathrm{C}$ ) to initiate OGD for $2 \mathrm{~h}$, and subsequently incubated in a $95 \% \mathrm{O}_{2} / 5 \% \mathrm{CO}_{2}$-mixed medium with high glucose for $24 \mathrm{~h}$. The cells were harvested for subsequent experiments.

\section{Neurological deficit scores}

The neurological deficits of animals were assessed and scored in accordance with the 5-point scale method of Nishimura et al. [23]. The evaluation criteria were as follows: 0 - normal score and no deficit, 1 - mild injury, the trunk and forelimbs were bent when the whole mouse was lifted by the tail, 2 - moderate neurological deficit, the contralateral of mouse was circled to the left, but the state of immobility was normal, 3 - severe neurological deficit, the mouse leaned to the contralateral side when resting, 4 - more serious neurological deficit, lack of spontaneous motor activity or unconsciousness.

\section{Measurement of infarct size}

After that, the mice were deeply anaesthetised and euthanised. Infarction size was evaluated and 
visualised using the 2,3,5-triphenyltetrazolium chloride (TTC; 603R302, Solarbio, Beijing, China) staining method. Brain slices $(1 \mathrm{~mm})$ were stained with $1 \%$ TTC at room temperature for 10-15 min and then photographed. The clearly visible red area was non-ischaemic, and the white area was infarcted. Then, Image J software was performed to quantify the size of cerebral infarction.

\section{Determination of brain oedema}

Brains were removed at $24 \mathrm{~h}$ post-reperfusion and weighed for wet weight. Then, they were dried and weighed. The degree of brain oedema was determined using the following formula: water content $(\%)=([$ wet weight - dry weight $]$ /wet weight $)$ $\times 100 \%[41]$.

\section{Haematoxylin and eosin staining}

Brain tissues collected were fixed and embedded. After deparaffinisation and rehydration, the sections of $5 \mu \mathrm{m}$ were stained with haematoxylin (H8070, Solarbio) and eosin (A600190, Sangon, Shanghai, China). The mounted slides were photographed using a DP73 Olympus microscope at 200x and $400 \times$ magnification.

\section{Nissl staining}

The $5 \mu \mathrm{m}$ brain slices were then set for Cresyl violet staining. Morphological alteration of CA1 in hippocampus was observed by the fluorescence microscope (200x and 400x). Finally, the number of Nissl bodies was counted from 5 randomly selected fields and the mean value was calculated.

\section{Measurement of ROS, MDA, SOD, and ATP}

Reactive oxygen species, malondialdehyde (MDA), SOD, and ATP levels in brain tissues and cultured neurons were assessed and quantified using the appropriate reagent kits following the instructions of Nanjing Jiancheng Bioengineering Institute.

\section{MitoSOX Red staining}

After $24 \mathrm{~h}$ of OGD/R induction, the mitochondrial superoxide levels in primary cultured hippocampal neurons were measured with MitoSOX Red reagent (M36008, MKBio, Shanghai, China), and photographed under a 400× fluorescence microscope.

\section{Determination of mitochondrial membrane potential $(\Delta \Psi \mathrm{m})$}

$\Delta \Psi \mathrm{m}$ in hippocampus and primary cultured hippocampal neurons was determined by the recommended assay kit (C2006, Beyotime, Shanghai, China; M8650, Solarbio). The samples were incubated (JC-1 staining solution) and analysed. The results were observed using fluorescence microscopy.

\section{Western blotting analysis}

Protein extractions were quantified using BCA assay. The separation of specified proteins was achieved by SDS-PAGE (P0015, Beyotime), and the proteins were electrotransferred to a PVDF membrane (IPVH00010, Millipore, Billerica, USA). After blocking with $5 \%(\mathrm{M} / \mathrm{V})$ nonfat dry milk, the membranes were incubated with primary antibodies against cytochrome c, Bax, Bcl-2, cleaved caspase-9, cleaved caspase-3, p38, p-p38, COX IV and $\beta$-actin at $4^{\circ} \mathrm{C}$. On the second day, the bands were incubated with horseradish peroxidase (HRP)-labelled secondary antibodies and visualised by an enhanced chemiluminescence system (ECL, P0018, Beyotime). Image analysis of protein blots was measured by the Gel-Pro-Analyzer software. The information of antibodies is described in Table I.

\section{TUNEL-NeuN staining}

Localisation of apoptotic neurons in brain tissues was measured by the fluorescent TUNEL/NeuN method [11]. Briefly, the slices were pretreated with Triton X-100 (ST795, Beyotime) and incubated in the TUNEL reaction mixture (11684817910, Roche, Basel, Switzerland) at room temperature. Sections were then cultured with NeuN primary antibody (ab104224, 1 : 400, Abcam, Cambridge, UK) overnight in a humid chamber, followed by the incubation of secondary antibody (A0521, Beyotime). Finally, the sections were counterstained with DAPI (C1002, Biosharp, Hefei, China) and observed using a DP73 microscopy at 200x and 400× magnification. The number of surviving neurons and apoptotic cells were counted, respectively.

\section{TUNEL staining}

Apoptotic neurons were measured with In Situ Cell Death Detection Kit (11684795910, Roche) in accordance with the description recommended by 
Table I. Antibody information and incubation conditions

\begin{tabular}{|c|c|c|c|c|c|c|c|}
\hline $\begin{array}{l}\text { Primary } \\
\text { antibodies }\end{array}$ & $\begin{array}{l}\text { Dilution } \\
\text { ratio }\end{array}$ & $\begin{array}{l}\text { Incubation } \\
\text { condition }\end{array}$ & Secondary antibodies & $\begin{array}{l}\text { Dilution } \\
\text { ratio }\end{array}$ & $\begin{array}{l}\text { Incubation } \\
\text { condition }\end{array}$ & Manufacturer & Number \\
\hline Bcl-2 antibody & $1: 1000$ & $4^{\circ} \mathrm{C}$ overnight & anti-rabbit IgG-HRP & $1: 5000$ & $37^{\circ} \mathrm{C} 45 \mathrm{~min}$ & CST & \#3498 \\
\hline Bax antibody & $1: 1000$ & $4^{\circ} \mathrm{C}$ overnight & anti-rabbit IgG-HRP & $1: 5000$ & $37^{\circ} \mathrm{C} 45 \mathrm{~min}$ & CST & \#5023 \\
\hline $\begin{array}{l}\text { Cleaved caspase-3 } \\
\text { antibody }\end{array}$ & $1: 1000$ & $4^{\circ} \mathrm{C}$ overnight & anti-rabbit IgG-HRP & $1: 5000$ & $37^{\circ} \mathrm{C} 45 \mathrm{~min}$ & CST & \#9661 \\
\hline $\begin{array}{l}\text { Cleaved caspase-9 } \\
\text { antibody }\end{array}$ & $1: 500$ & $4^{\circ} \mathrm{C}$ overnight & anti-rabbit IgG-HRP & $1: 5000$ & $37^{\circ} \mathrm{C} 45 \mathrm{~min}$ & CST & \#9509 \\
\hline p-p38 antibody & $1: 1000$ & $4^{\circ} \mathrm{C}$ overnight & anti-rabbit IgG-HRP & $1: 5000$ & $37^{\circ} \mathrm{C} 45 \mathrm{~min}$ & CST & \#4511 \\
\hline p38 antibody & $1: 2000$ & $4^{\circ} \mathrm{C}$ overnight & anti-rabbit IgG-HRP & $1: 5000$ & $37^{\circ} \mathrm{C} 45 \mathrm{~min}$ & CST & \#9212 \\
\hline $\begin{array}{l}\text { Cytochrome c } \\
\text { antibody }\end{array}$ & $1: 1000$ & $4^{\circ} \mathrm{C}$ overnight & anti-rabbit IgG-HRP & $1: 5000$ & $37^{\circ} \mathrm{C} 45 \mathrm{~min}$ & CST & \#11940 \\
\hline
\end{tabular}

the manufacturer. The slices were treated with $0.1 \%$ Triton X-100 (ST795, Beyotime) and then stained by TUNEL staining followed by DAPI counterstaining (D106471-5 mg, Aladdin). The apoptotic neurons were counted from $400 \times$ microscopic field in each section.

\section{Statistical analysis}

Data of all at least three samples were expressed as mean \pm standard deviation (SD). Their difference analysis was shown using GraphPad Prism 8.0 software. Generally speaking, Student's $t$-tests were used to analyse the differences between pairs, and one-way analysis of variance (ANOVA) was performed for statistical significance of difference among groups. $P$-values $<0.05$ were considered statistically significant.

\section{Results}

\section{Amifostine restored brain functional outcome in MCAO/R mice}

To reveal the positive effect of amifostine on I/R injury in brain, we observed and analysed the brain functional outcome after reperfusion. The neurological deficit scores of the mice were significantly increased after reperfusion. Strikingly, amifostine treatment markedly reduced neurological deficits and appeared to be dose-dependent (Fig. 1A). Next, TTC staining revealed the brain infarct volume after injury, and the areas of ischaemic necrosis were stained white (Fig. 1B). The percentage of brain infarct area was obviously enlarged in the MCAO/R group, while amifostine treatment apparently diminished it, particularly in the MCAO/R $+\mathrm{H}$ group (Fig. 1C).
Also, there was a significant increase in brain water content of MCAO/R mice, which could be relieved by amifostine treatment (Fig. 1D). Furthermore, $H \& E$ staining displayed the histopathological and morphological alteration of hippocampus CA1 (Fig. $1 \mathrm{E})$. In the sham group, the neuron cell structure was normal, the edge was clear, and the nuclei and intercellular substance were stained evenly. In contrast, the neurons in the MCAO/R group exhibited a disordered arrangement with obvious oedema and necrosis. Importantly, treatment of amifostine significantly attenuated the extent of brain oedema and neuronal necrosis. By using Nissl staining, the results showed that the number of stained Nissl's bodies was significantly declined in mice of the MCAO/R group. After amifostine treatment, its number was increased significantly (Fig. 1F). Our findings provided evidence that amifostine could prevent brain failure caused by I/R.

\section{Amifostine attenuated \\ MCAO/R-induced oxidative stress and mitochondrial damage}

To determine the effect of amifostine on oxidative stress and mitochondrial damage, we performed subsequent experiments. As demonstrated in Figure $2 A-C, E, I / R$ injury significantly induced ROS and MDA levels, and reduced SOD activity and ATP level. Using flow cytometry analysis, we found a obvious decline in fluorescence intensity in the hippocampus of MCAO/R mice, indicating the depolarization of mitochondria (Fig. 2D). Western blotting analysis demonstrated that I/R injury upregulated cytochrome $\mathrm{c}$ expression in cytoplasm and 


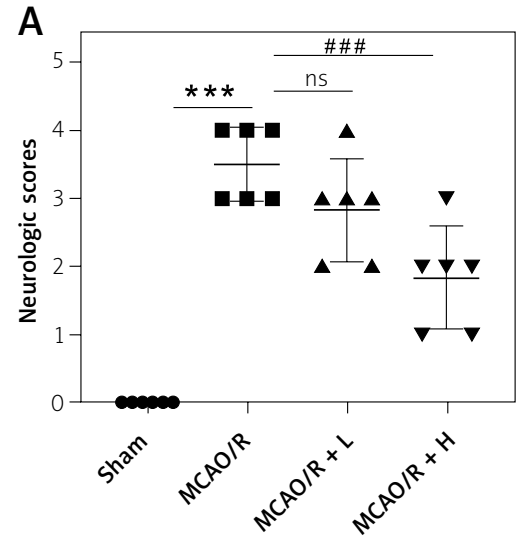

B

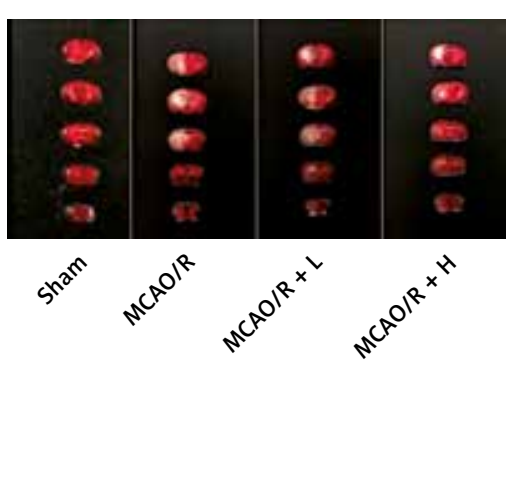

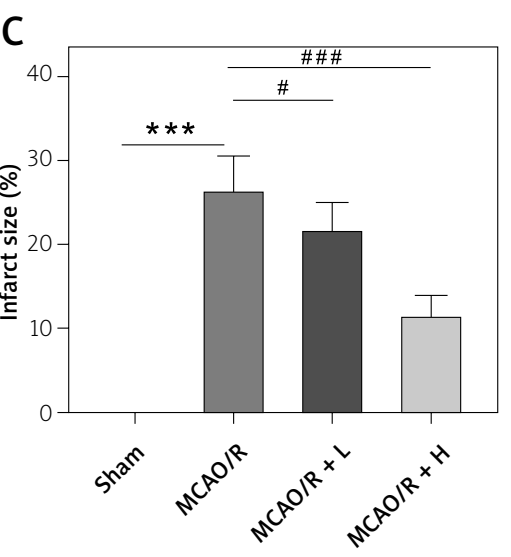

$E$

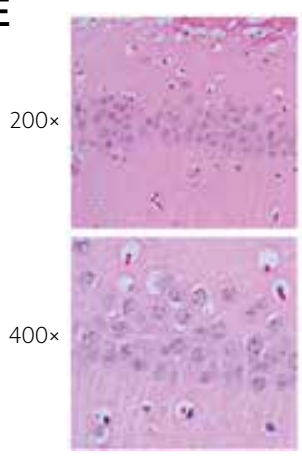

Sham

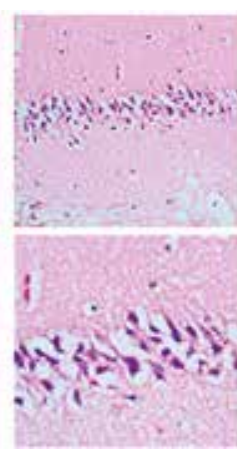

MCAO/R
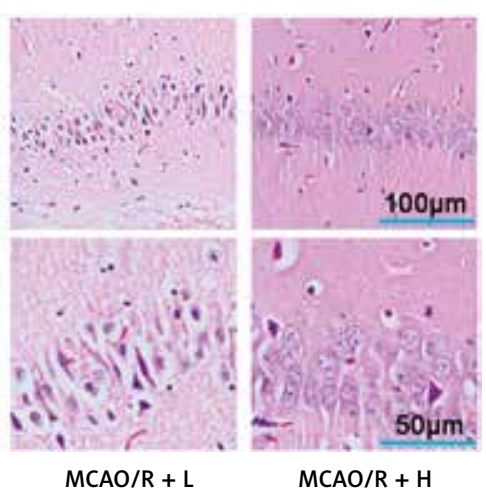

$\mathrm{F}$
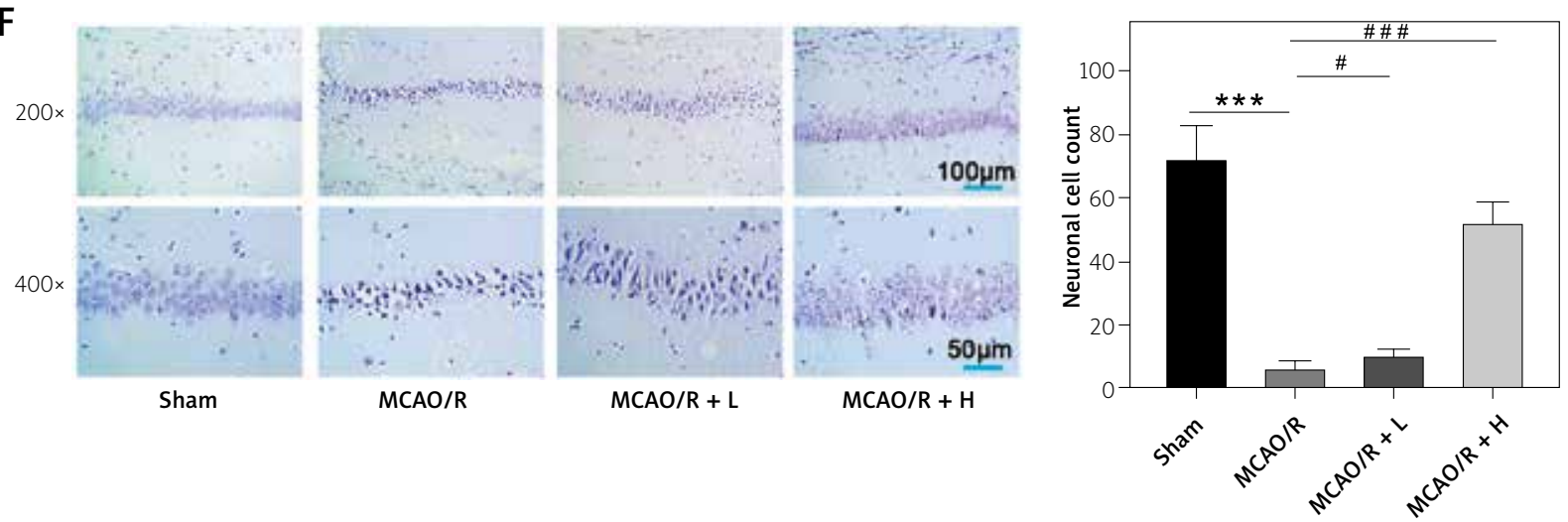

Fig. 1. Amifostine protected against ischaemia and reperfusion injury in brain. Male C57BL/6 mice were intraperitoneally injected with amifostine (200 mg/kg or $400 \mathrm{mg} / \mathrm{kg}$ ) before MCAO/R surgery. A) Assessment of neurological deficit scores at $24 \mathrm{~h}$ after reperfusion. B, C) TTC staining was used to evaluate the cerebral infarct area, followed by quantification of infarct volumes. D) Brain water content $=$ ([wet weight dry weight]/wet weight) $\times 100$. E) H\&E staining showed the pathological alterations of neurons in hippocampus CA1 region. F) Nissl staining revealed the surviving neurons in hippocampus, followed by quantification. ${ }^{{ }^{*}} p<0.01,{ }^{\star \star \star} p<0.001$ vs. sham group; ${ }^{\#} p<0.05,{ }^{\# \# \# p}<0.001$ vs. MCAO/R group. 
A

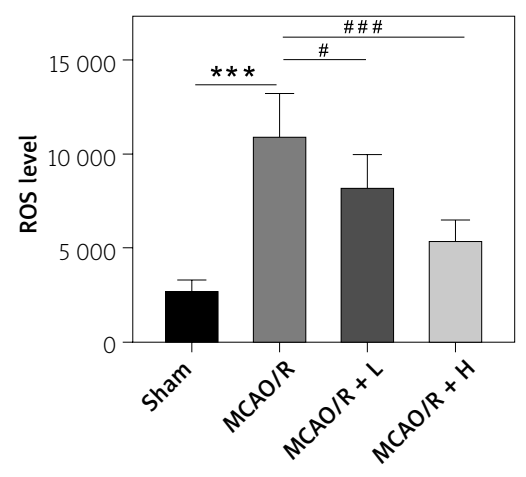

D
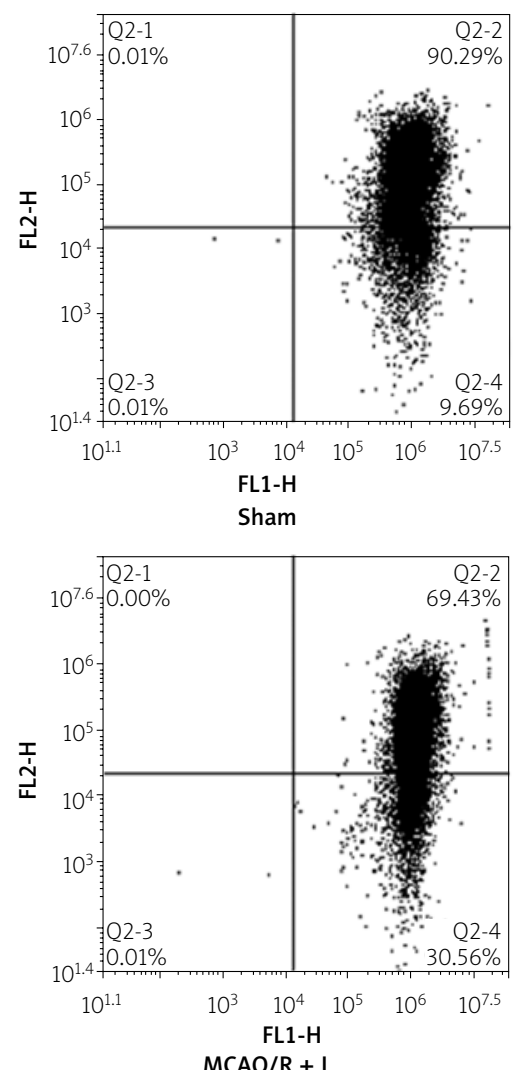

B
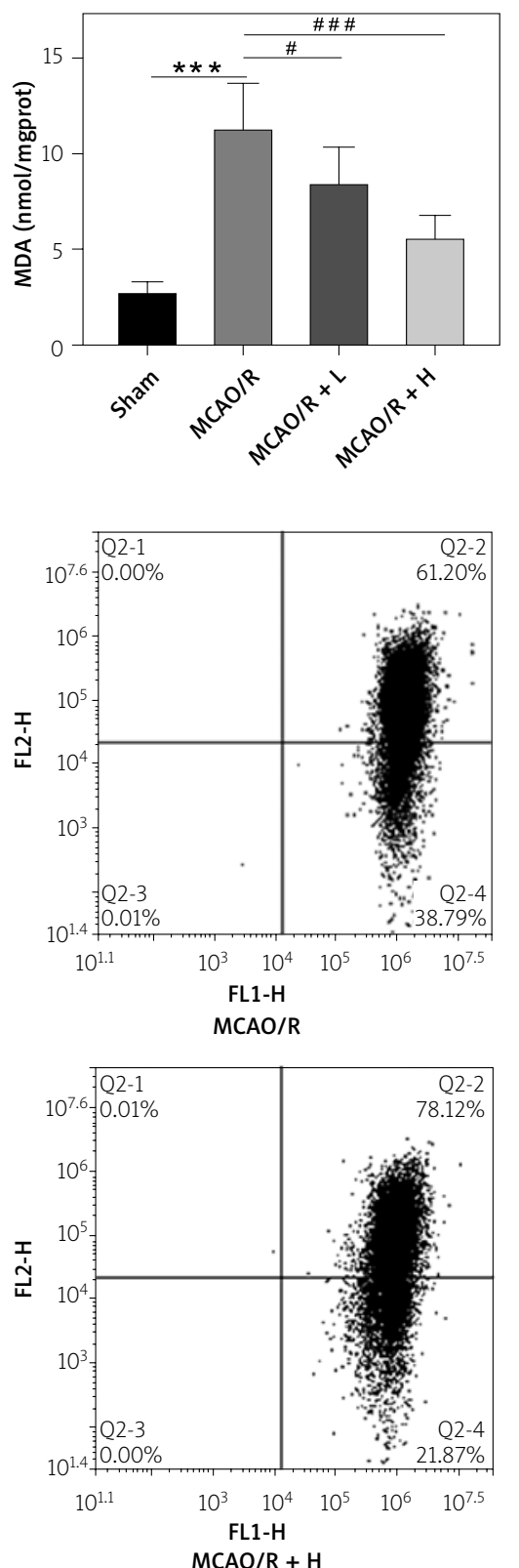

C
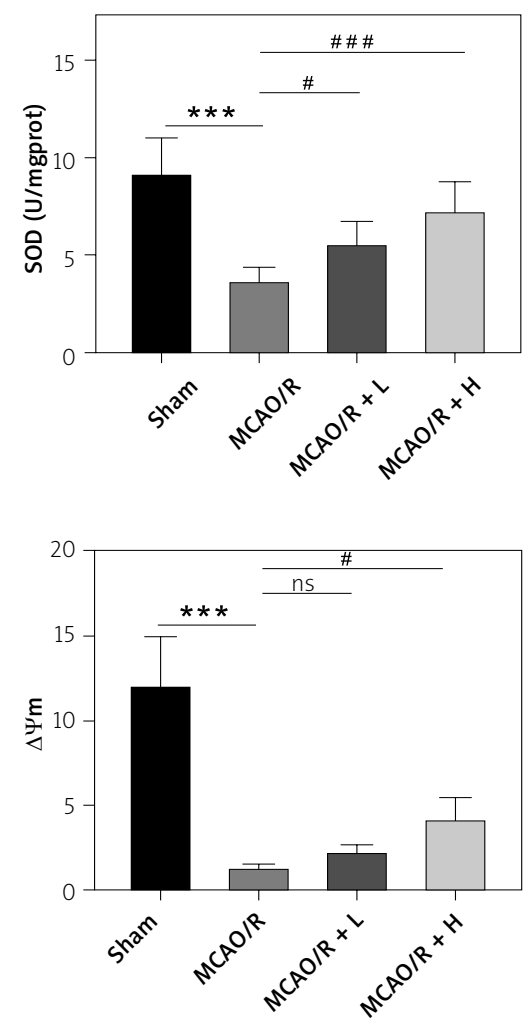

$\mathrm{E}$

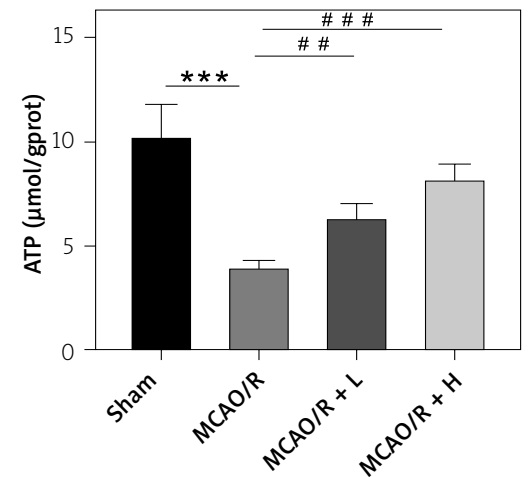

$\mathbf{F}$

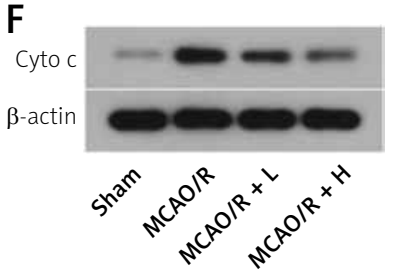

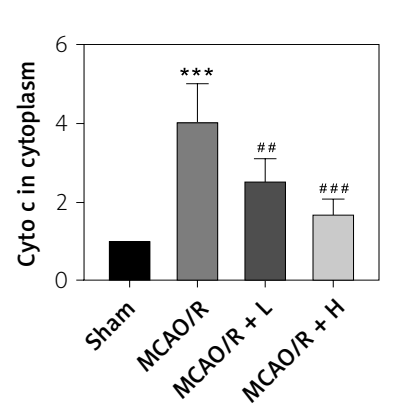

G

Cyto c

COXIV

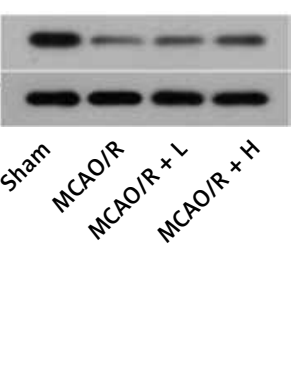

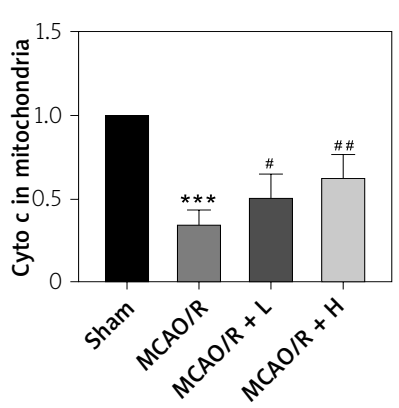

Fig. 2. Amifostine attenuated oxidative and mitochondrial damage after reperfusion. A-C) At $24 \mathrm{~h}$ post-reperfusion, the ROS level, MDA content and SOD activity in hippocampus were measured by the kits. D) Mitochondrial membrane potential changes $(\Delta \Psi \mathrm{m})$ in hippocampus were determined using flow cytometry. E) ATP level was determined by the ATP assay kit. F, G) Cytochrome $c$ (Cyto c) expression levels in cytoplasm and mitochondria were analyzed using western lightning plus-ECL. ${ }^{* * *} p<0.001$ vs. Sham group; ${ }^{*} p<0.05$, $\# \#<0.01, \# \# p<0.001$ vs. MCAO/R group. 
downregulated cytochrome $c$ in mitochondria (Fig. $2 \mathrm{~F}, \mathrm{G})$. However, amifostine treatment obviously diminished the above-mentioned results induced by $M C A O / R$, indicating that amifostine could attenuate I/R-caused mitochondrial damage in the brain.

\section{Amifostine inhibited MCAO/R-induced apoptosis}

Apoptosis is the ultimate and major determinant of brain damage by I/R. Here, TUNEL/NeuN staining revealed that MCAO/R induced a significant decrease in the number of surviving neurons but an increase in the number of apoptotic cells. After treatment of amifostine, the apoptotic cells were dose-dependently decreased (Fig. 3A). Furthermore, protein expression of genes related to apoptosis was estimated by western blotting analysis (Fig. 3B, C). MCAO/R induced the upregulation of Bax, cleaved caspase- 9 , and cleaved caspase- 3 levels but downregulation of $\mathrm{Bcl}-2$. In terms of p38 signalling transduction, MCAO/R increased the p-p38 level but had no impact on p38. Nevertheless, compared with the MCAO/R group, amifostine treatment in part reversed MCAO/R-induced apoptosis and p38 signalling pathway activation. These results showed that amifostine suppressed brain I/R injury-induced neuronal loss, possibly modulated by the p38 signalling pathway.

\section{Amifostine alleviated OGD/R-induced oxidative stress and mitochondrial damage}

Based on the protective effect of amifostine against $M C A O / R$ in vivo, we further verified its in vitro effect on mouse hippocampal neurons. After OGD/R induction, ROS and mitochondrial superoxide levels were increased, but total SOD, SOD1 (Cu/Zn SOD, cytoplasmic SOD), and SOD2 (mitochondrial SOD) activities were decreased. Treatment of amifostine could obviously alleviate the above oxidative stress markers in OGD/R-treated neurons (Fig. 4A-C). In addition, the depolarisation of $\Delta \Psi \mathrm{m}$ was visualised through a JC-1 fluorescent probe. In general, red fluorescence image presented polarised mitochondria with high $\Delta \Psi \mathrm{m}$, and green fluorescence indicated depolarised mitochondria with low $\Delta \Psi \mathrm{m}$. JC-1 staining showed that in the control group, the red fluorescence was obvious and the green fluorescence was relatively weak, suggesting a relatively stable $\Delta \Psi \mathrm{m}$ and normal cellular function. In the OGD/R group, the ratio of red and green fluorescence was significantly decreased, suggesting the obvious decrease in $\Delta \Psi \mathrm{m}$. However, amifostine treatment showed enhanced red fluorescence and reduced green fluorescence, indicating that the $\Delta \Psi \mathrm{m}$ was restored to a stable state after amifostine treatment (Fig. 4D). These findings confirmed the antioxidant activity of amifostine in cerebral I/R injury.

\section{Amifostine alleviated OGD/R-induced neuronal apoptosis}

TUNEL staining was carried out for apoptosis analysis $48 \mathrm{~h}$ after OGD/R induction. Accordingly, exposure to OGD/R contributed to neuronal apoptosis, which was partially reversed by amifostine pretreatment (Fig. 5A). Moreover, western blotting assay showed that $\mathrm{OGD} / \mathrm{R}$ induced an increase in Bax, cleaved caspase- 9 , cleaved caspase-3, and p-p38 and a decrease in $\mathrm{Bcl}-2$. However, these changes induced by OGD/R were blocked by amifostine pretreatment (Fig. 5B, C).

\section{Discussion}

Amifostine has been recognised as an effective cytoprotectant drug that plays a role in the prevention of I/R damage [39]. This current work is the first to reveal the role of amifostine in cerebral I/R injury, from aspects of oxidative stress, mitochondrial dysfunction, and apoptosis. In our current study, we demonstrate that amifostine can repair neurological deficits, and reduce infarct area and brain oedema. Moreover, amifostine reduces I/R-induced neuron loss via reducing overactive oxidative stress and mitochondria damage. These results indicate the beneficial effect of amifostine in the treatment of ischaemic injury in the brain.

Accumulating research has demonstrated that oxidative stress is the key factor in the pathogenesis of multiple neurodegenerative diseases [3,33]. During I/R injury, excessive oxygen free radicals and ROS will be produced, subsequently activating multiple signals, and leading to the dysfunction of lipids, mitochondria, and other cellular components [16,37]. In the antioxidant system, SODs such as SOD1 and SOD2 are considered as superoxide radical scavengers to prevent ROS attack [1]. MDA is identified as a marker of oxidant stress, indicating the content of lipid peroxidation. High lipid levels and oxygen consumption are responsible for oxidative damage in the brain [25]. Amifostine has been found to scavenge 
A
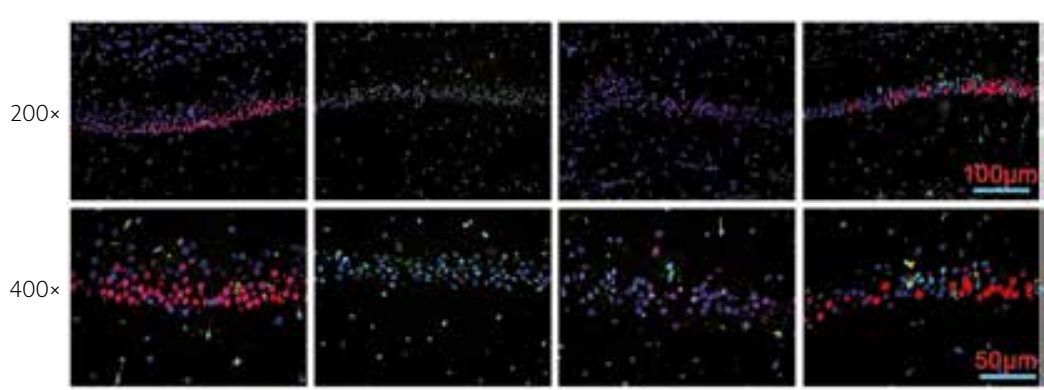

MCAO/R

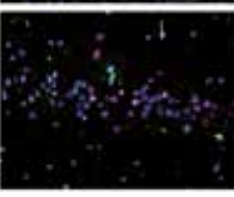

$M C A O / R+L$

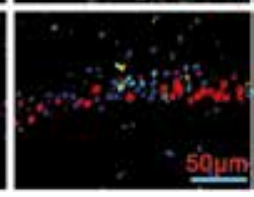

$M C A O / R+H$
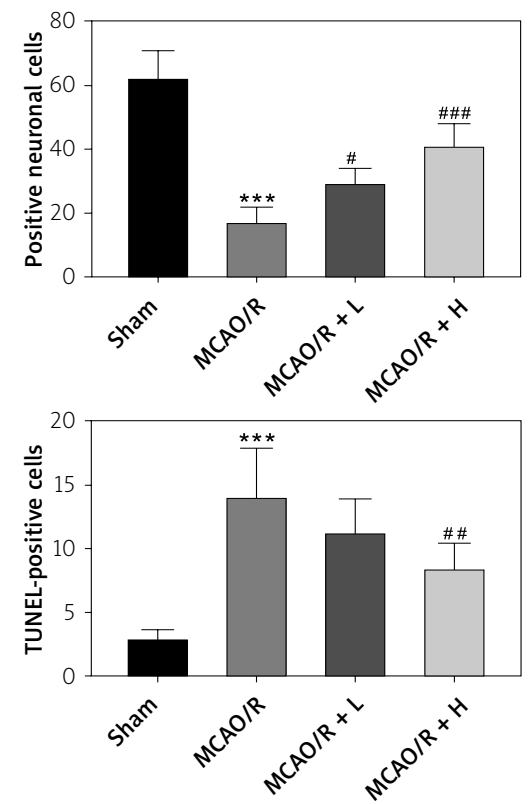

B
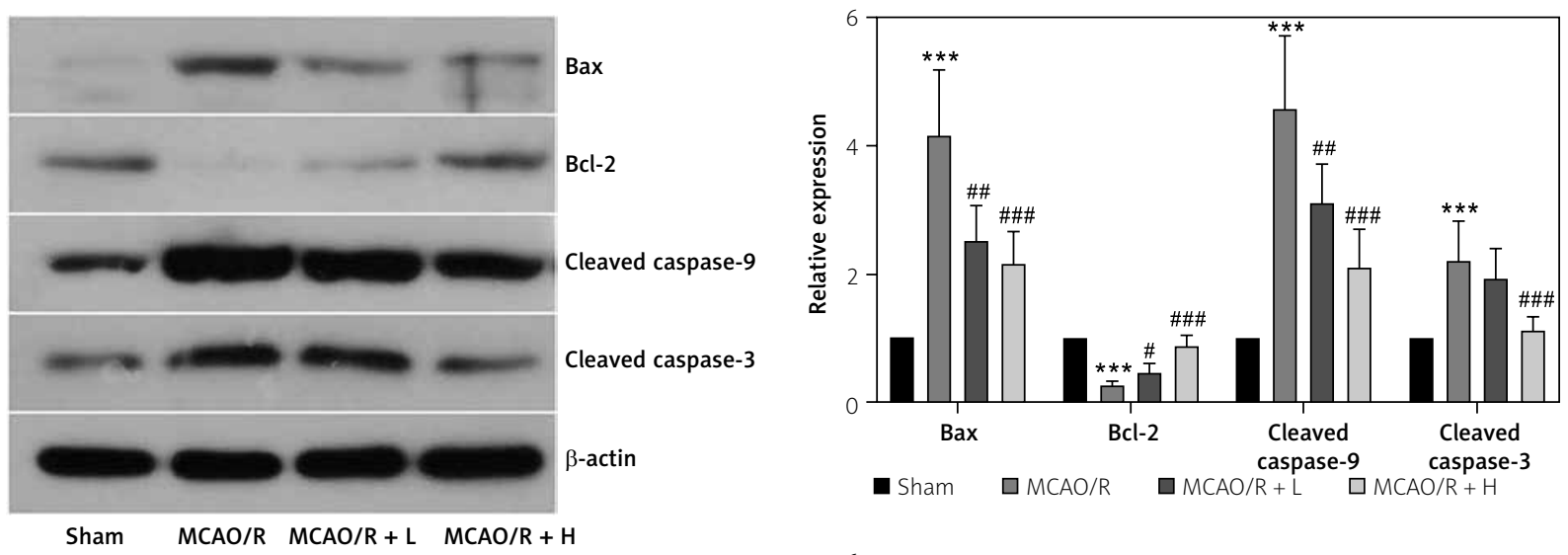

C
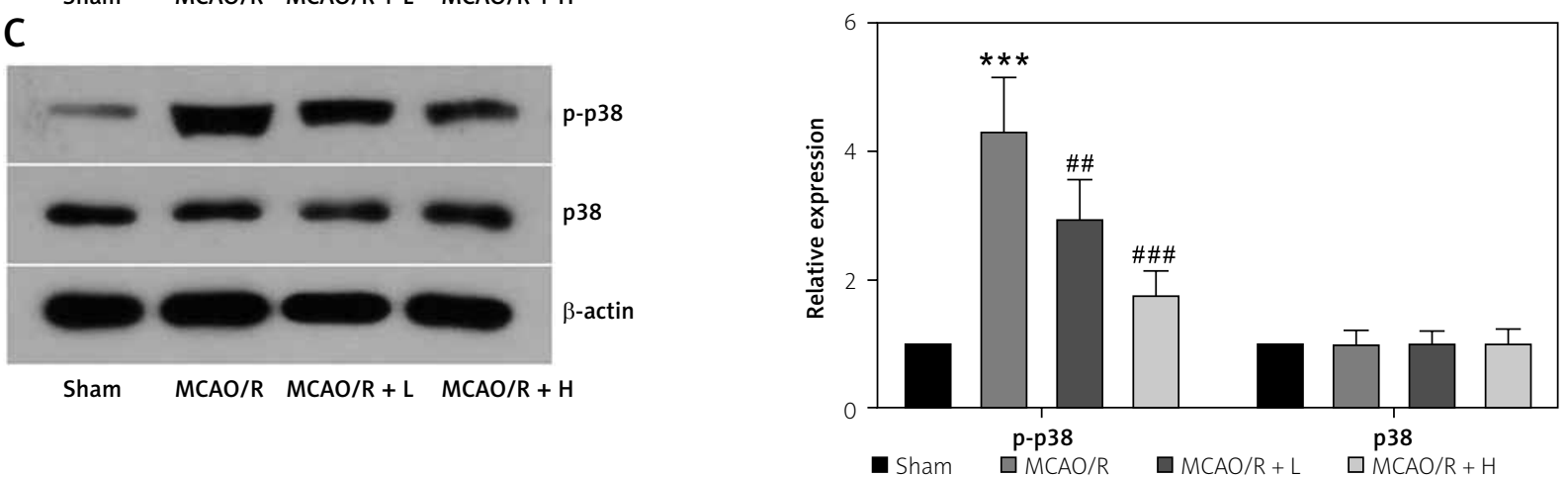

Fig. 3. Amifostine inhibited neuronal apoptosis $24 \mathrm{~h}$ after reperfusion. A) TUNEL-immunofluorescence assay represented the distribution of neurons and apoptotic cells in hippocampus CA1 region at magnification 200x and 400x. B, C) Western blot assay was carried out to detect levels of Bax, Bcl-2, cleaved caspase-9,

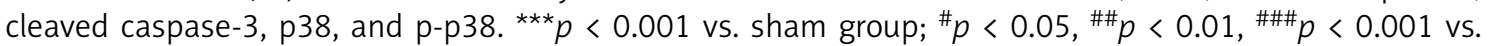
$\mathrm{MCAO/R}$ group. 

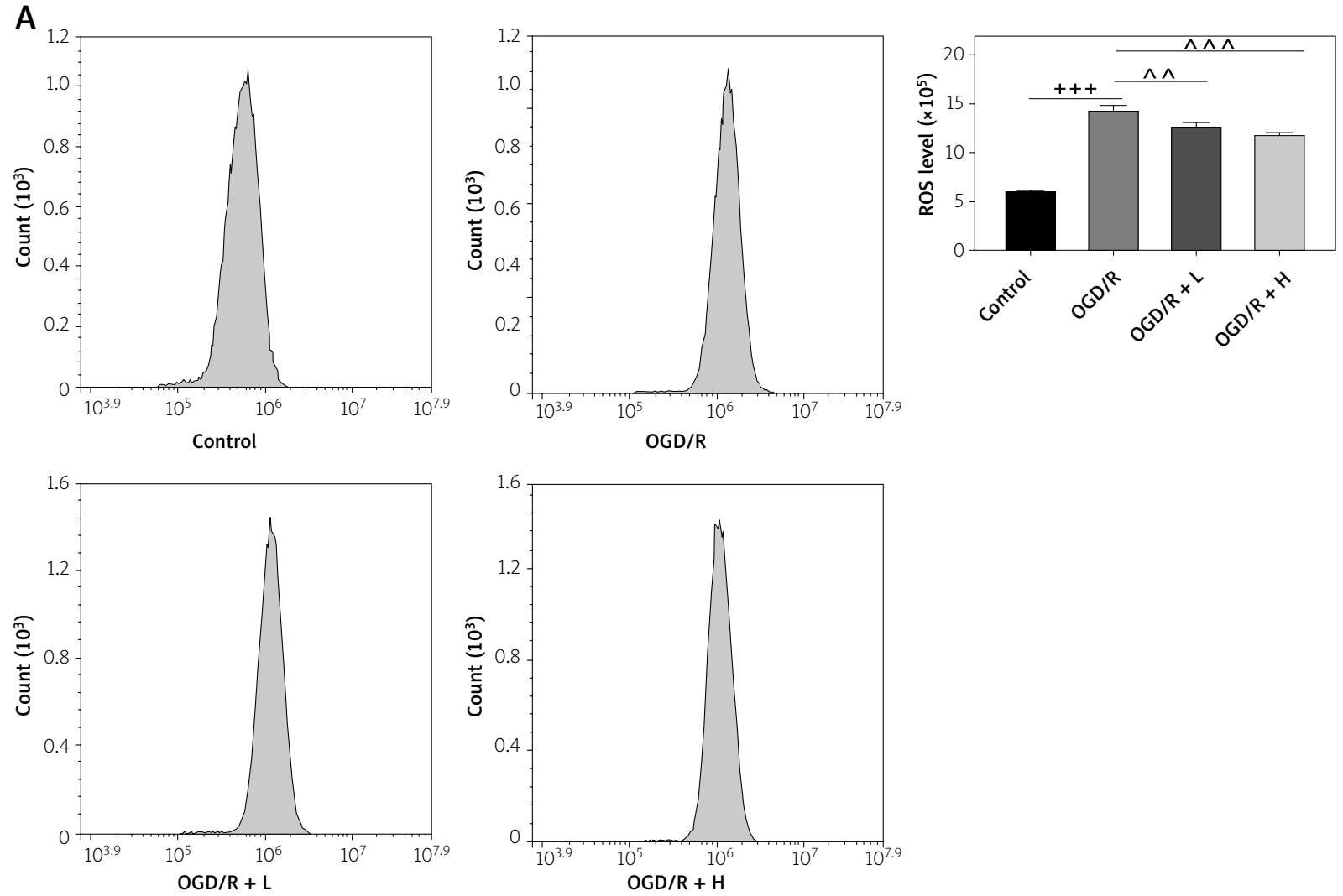

B
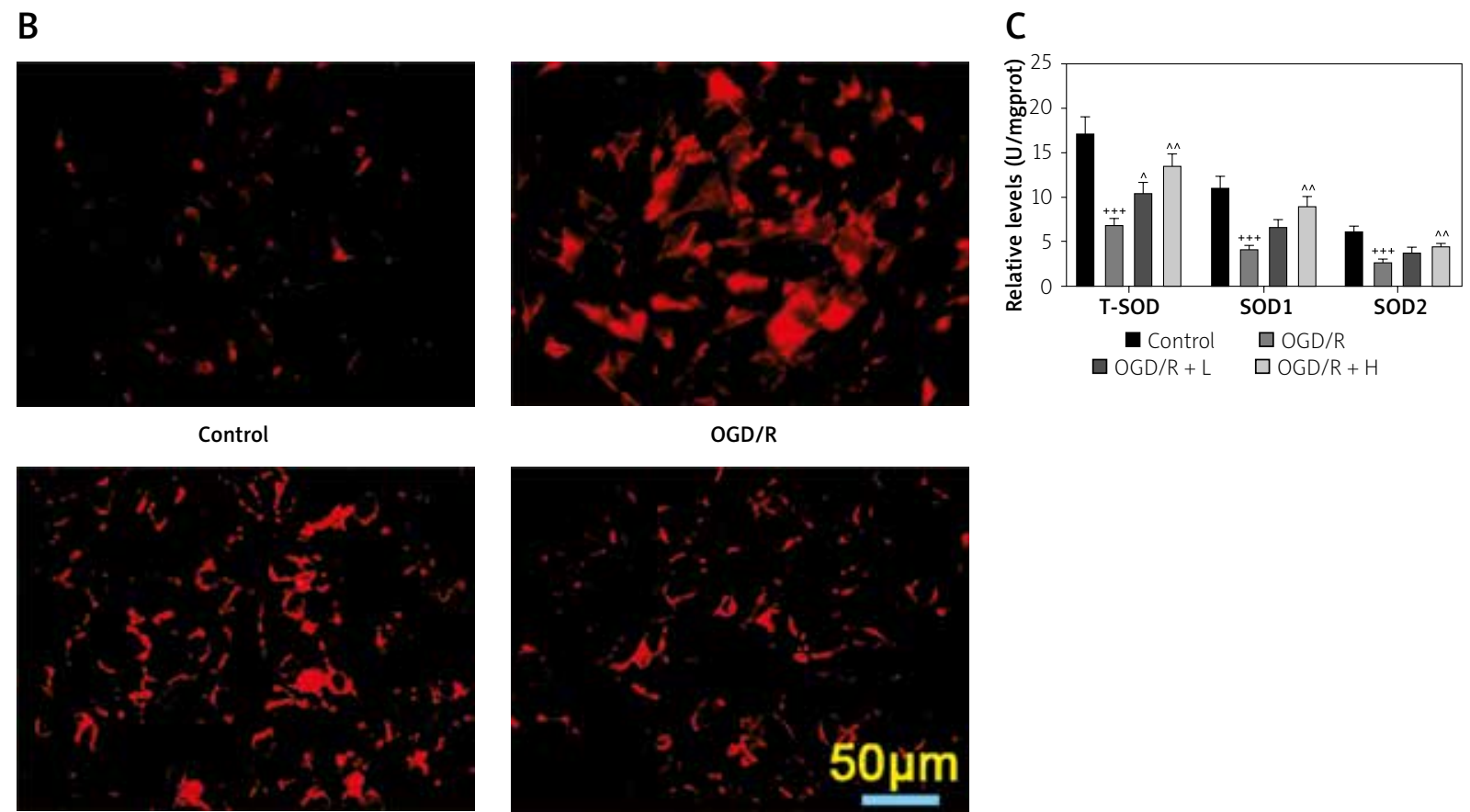

$\mathrm{OGD} / \mathrm{R}+\mathrm{L}$

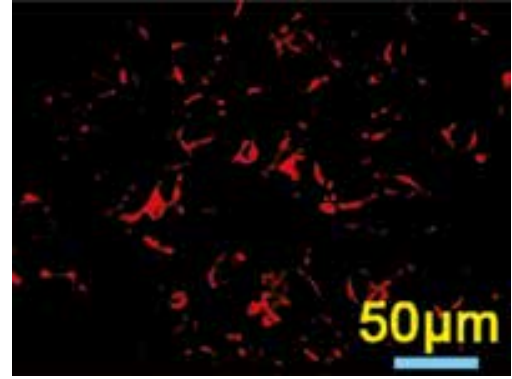

$\mathrm{OGD} / \mathrm{R}+\mathrm{H}$

Fig. 4. Amifostine alleviated $O G D / R$-induced oxidative and mitochondrial damage. After pre-treatment of amifostine for $30 \mathrm{~min}$, the primary cultured mouse hippocampal neurons underwent OGD/R to induce neuronal cell injury. A) Flow cytometry was performed to detect ROS level. B) MitoSOX Red staining revealed the production of mitochondrial superoxide. C) SOD assay kits were used for the determination of the total SOD, cytoplasmic SOD (SOD1) and mitochondrial SOD (SOD2) activities. ${ }^{+++} p<0.001 \mathrm{vs.} \mathrm{Control} \mathrm{group;}$ $\wedge p<0.05, \wedge \wedge p<0.01, \wedge \wedge \wedge p<0.001$ vs. OGD/R group. 
D

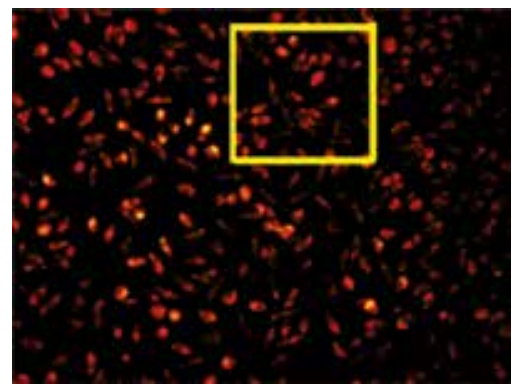

Control

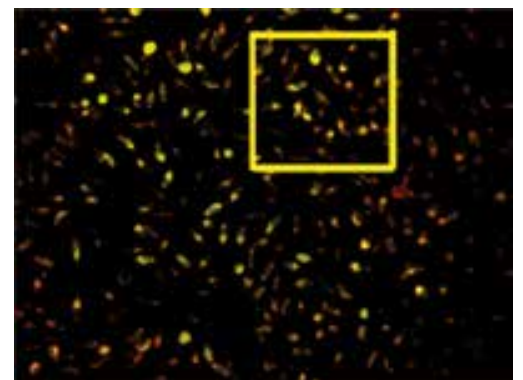

$\mathrm{OGD} / \mathrm{R}+\mathrm{L}$
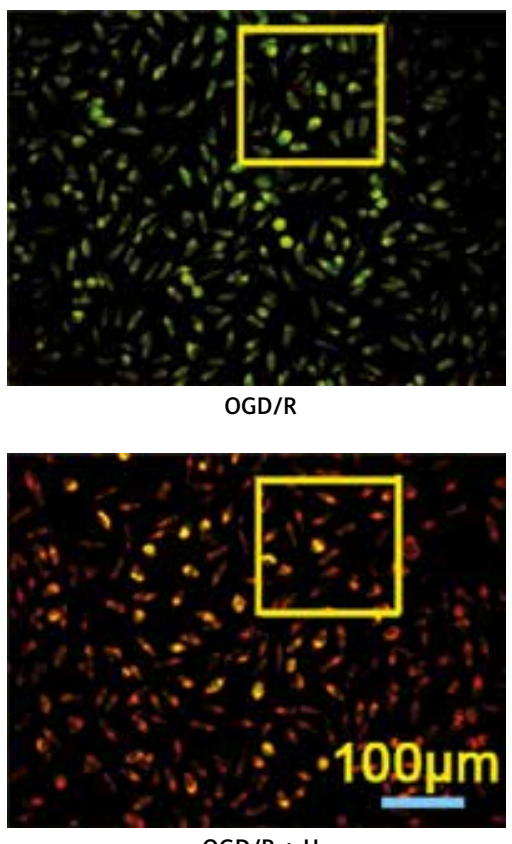

$\mathrm{OGD} / \mathrm{R}+\mathrm{H}$

Fig. 4. D) JC-1 fluorescent staining revealed the high or low mitochondrial membrane potential $(\Delta \Psi \mathrm{m})$ in hippocampal neurons.

ROS, induce antioxidant enzymatic activities, and reduce oxidative stress in spinal cord and heart after I/R injury [7,39]. In this study, amifostine significantly attenuates the increase of ROS level and MDA content, and the decrease of SOD activity in MCAO/Rinduced hippocampal tissues and OGD/R-induced primary cultured hippocampal neurons, which is consistent with the previous results on I/R injury.

Mitochondria form a complex, dynamic, interconnected network that exerts a variety of biological functions. Mitochondrial dysfunction is a common predictor of many human diseases, such as cancer, metabolic disorders, and neurodegenerative diseases [24]. $\Delta \Psi \mathrm{m}$ is a necessary component that can selectively eliminate dysfunctional mitochondria to maintain mitochondrial homeostasis. Longterm increase or decrease of $\Delta \Psi \mathrm{m}$ may contribute to various pathologies [45]. In addition, abnormalities in ATP generation are thought to be a core of mitochondrial dysfunction. Impaired mitochondrial function contributes to the reduction of ATP production [6]. Relatively stable $\Delta \Psi \mathrm{m}$ and ATP level are vital for the maintenance of normal cell function. Moreover, cytochrome c, an important mitochondrial molecule, exhibits peroxidase activity and functions as a scav- enger of ROS following neuronal damage [4]. More importantly, previous studies revealed that amifostine induces antioxidant enzymatic activities, and reduces mitochondrial damage and neuronal cell death $[12,14]$. In line with these observations, we find that the decreased $\Delta \Psi \mathrm{m}$ and ATP level caused by I/R injury are partially abolished by amifostine treatment. Amifostine treatment also significantly prevents cytochrome c escaping into cytoplasm, along with the increase of cytochrome $c$ in mitochondria. Increasing evidence demonstrates that there are some phenomena, including stable $\Delta \Psi \mathrm{m}$, elevated ATP level, and accumulated cytochrome $c$ in mitochondria, indicating improvement of I/R brain injury $[8,38]$. Overall, these findings indicate that amifostine attenuates brain I/R injury via attenuating oxidative stress response and mitochondrial dysfunction.

Furthermore, ROS overproduction can activate mitochondrial apoptotic pathway, eventually leading to cell death [44]. Inhibition of apoptosis is the key step for the therapy of ischaemic stroke [34]. It is generally known that the ratio of anti-apoptotic proteins to pro-apoptotic proteins is crucial for the modulation of cellular survival/death. $\mathrm{Bcl}-2$, an anti-apoptotic protein, can act as an effective free 
A

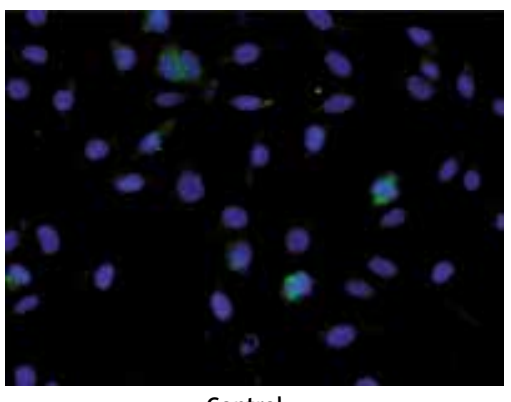

Control

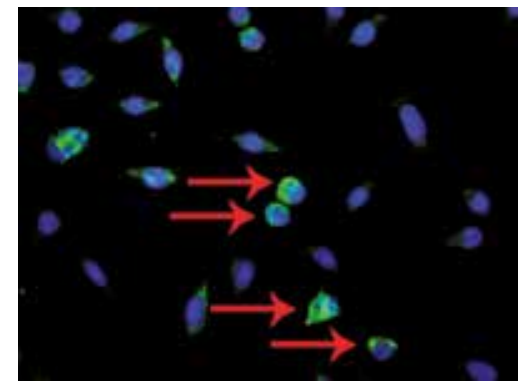

$\mathrm{OGD} / \mathrm{R}+\mathrm{L}$

C

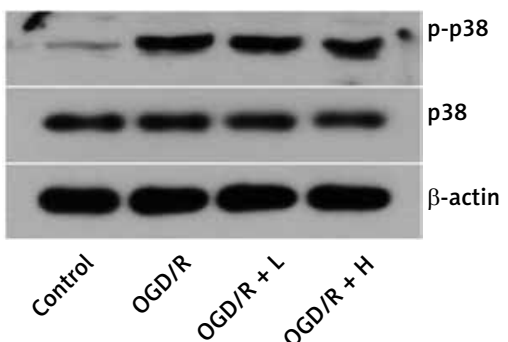

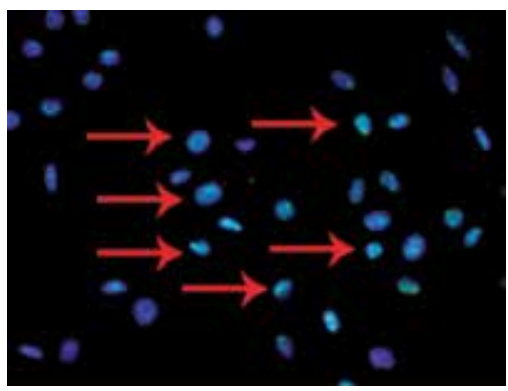

OGD/R

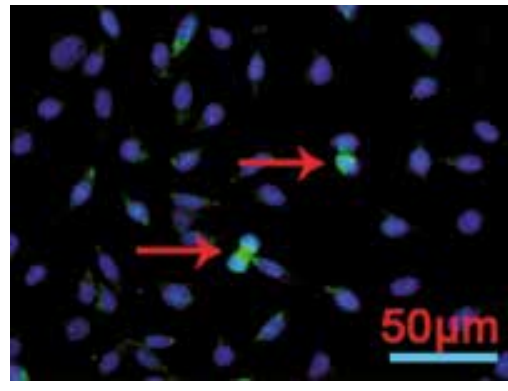

$\mathrm{OGD} / \mathrm{R}+\mathrm{H}$

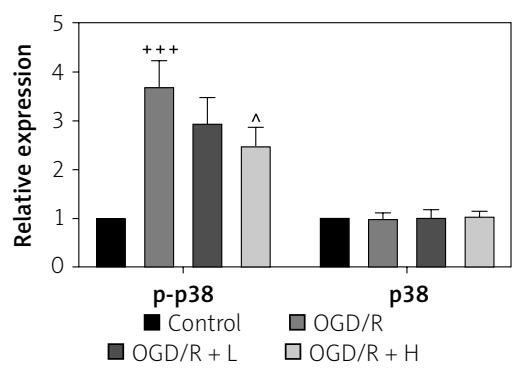

B
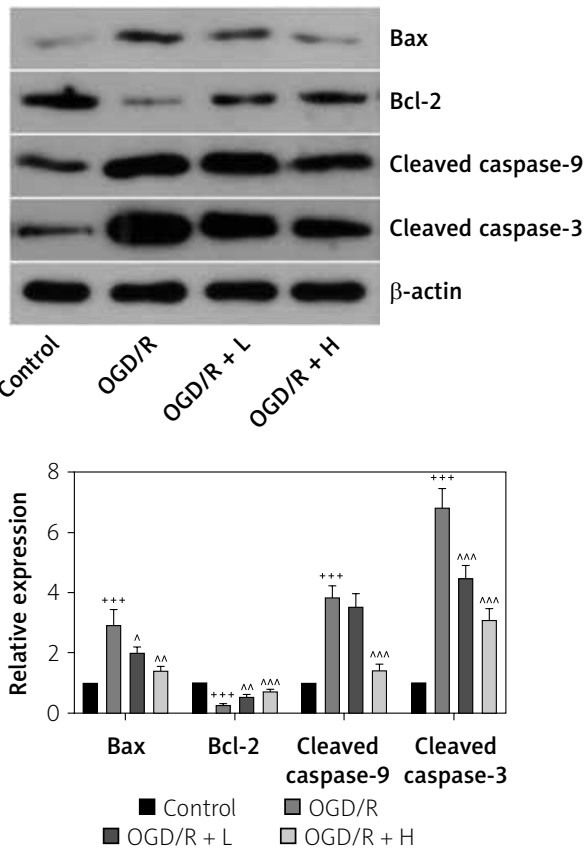

Fig. 5. Amifostine suppressed OGD/R-induced neuronal apoptosis. A) Cell apoptosis was determined by TUNEL staining. B, C) Relative levels of Bax, Bcl-2, cleaved caspase-9, cleaved caspase-3, p38 and p-p38 were measured through western blot analysis. ${ }^{+++} p<0.001$ vs. control group; $\wedge p<0.05, \wedge \wedge p<0.01, \wedge \wedge \wedge p<0.001$ vs. OGD/R group.

radical scavenger to eliminate excessive ROS generation [26]. Conversely, Bax is a pro-apoptotic protein that can cause $\Delta \Psi \mathrm{m}$ loss and the release of cytochrome c [42]. Then, cytochrome c in the cytoplasm induces Apaf-1 activation and forms apoptosome of cytochrome C/Apaf-1/pro-caspase-9. It can cleave pro-caspase- 9 into cleaved caspase- 9 and then cleave downstream pro-caspase 3 into cleaved caspase-3, thereby activating the apoptotic signalling pathway [29]. After brain I/R injury, pro-apoptotic proteins are highly expressed, while anti-apoptotic proteins are expressed lowly [36]. Our data show that OGD/R stimulates neuronal apoptosis, accompanied by the elevation of Bax, cleaved caspase-9, cleaved caspase-3, and the decline of $\mathrm{Bcl}-2$. Interest- ingly, amifostine treatment efficiently ameliorates apoptosis induced by OGD/R. In addition, a study has demonstrated that the anti-apoptotic effect of amifostine is regulated by p38 signalling pathway [31]. When extracellular stimuli occur, p38 can be phosphorylated and can activate transcription factors, ultimately initiating cell apoptosis. Amifostine markedly inhibits phosphorylated p38, thereby inhibiting cell death [31]. In our study, p-p38 expression was upregulated by $O G D / R$, while it was remarkably repressed by amifostine pretreatment, indicating that amifostine may suppress the activation of p38 apoptosis signalling. All the above data indicate that amifostine may suppress neuronal apoptosis by modulating the p38 signalling activation. 
In summary, our findings indicate that amifostine may serve a neuroprotective role in cerebral-neuron injury caused by I/R. Notably, this protective effect is probably realised by attenuating $\mathrm{p} 38$-associated oxidative stress, mitochondrial dysfunction, and neuronal apoptosis. All results indicate that amifostine can be used as a candidate agent for the treatment of cerebral I/R injury, providing a new insight for the therapy of I/R brain injury.

\section{Disclosure}

The authors report no conflict of interest.

\section{References}

1. Adibhatla RM, Hatcher JF. Lipid oxidation and peroxidation in CNS health and disease: from molecular mechanisms to therapeutic opportunities. Antioxid Redox Signal 2010; 12: 125-169.

2. Atalay H, Aybek H, Koseoglu M, Demir S, Erbay H, Bolaman AZ, Avci A. The effects of amifostine and dexamethasone on brain tissue lipid peroxidation during oxygen treatment of carbon monoxide-poisoned rats. Adv Ther 2006; 23: 332-341.

3. Attia GM, Elmansy RA, Elsaed WM. Neuroprotective effect of nilotinib on pentylenetetrazol-induced epilepsy in adult rat hippocampus: involvement of oxidative stress, autophagy, inflammation, and apoptosis. Folia Neuropathol 2019; 57: 146-160.

4. Bajwa E, Pointer CB, Klegeris A. The role of mitochondrial damage-associated molecular patterns in chronic neuroinflammation. Mediators Inflamm 2019; 2019: 4050796.

5. Chiang T, Messing RO, Chou WH. Mouse model of middle cerebral artery occlusion. J Vis Exp 2011; 48: 2761.

6. Chistiakov DA, Shkurat TP, Melnichenko AA, Grechko AV, Orekhov AN. The role of mitochondrial dysfunction in cardiovascular disease: a brief review. Ann Med 2018; 50: 121-127.

7. Chronidou F, Apostolakis E, Papapostolou I, Grintzalis K, Georgiou CD, Koletsis EN, Karanikolas M, Papathanasopoulos P, Dougenis D. Beneficial effect of the oxygen free radical scavenger amifostine (WR-2721) on spinal cord ischemia/reperfusion injury in rabbits. J Cardiothorac Surg 2009; 4: 50.

8. Dai Y, Zhang H, Zhang J, Yan M. Isoquercetin attenuates oxidative stress and neuronal apoptosis after ischemia/reperfusion injury via Nrf2-mediated inhibition of the NOX4/ROS/NF- $\mathrm{BB}$ pathway. Chem Biol Interact 2018; 284: 32-40.

9. Fletcher L, Evans TM, Watts LT, Jimenez DF, Digicaylioglu M. Rapamycin treatment improves neuron viability in an in vitro model of stroke. PLoS One 2013; 8: e68281.

10. Galkin A. Brain ischemia/reperfusion injury and mitochondrial complex I damage. Biochemistry (Mosc) 2019; 84: 1411-1423.

11. Garrido-Gil P, Belzunegui S, San Sebastián W, Izal-Azcárate A, López B, Marcilla I, Luquin MR. 1-Methyl-4-phenyl-1,2,3,6-tetrahydropyridine exposure fails to produce delayed degeneration of substantia nigra neurons in monkeys. J Neurosci Res 2009; 87: 586-597.

12. Grdina DJ, Murley JS, Kataoka Y, Baker KL, Kunnavakkam R, Coleman MC, Spitz DR. Amifostine induces antioxidant enzy- matic activities in normal tissues and a transplantable tumor that can affect radiation response. Int J Radiat Oncol Biol Phys 2009; 73: 886-896.

13. He X, Mo Y, Geng W, Shi Y, Zhuang X, Han K, Dai Q, Jin S, Wang J. Role of Wnt/ $\beta$-catenin in the tolerance to focal cerebral ischemia induced by electroacupuncture pretreatment. Neurochem Int 2016; 97: 124-132.

14. Jia J, Zhang L, Shi X, Wu M, Zhou X, Liu X, Huo T. SOD2 mediates amifostine-induced protection against glutamate in PC12 cells. Oxid Med Cell Longev 2016; 2016: 4202437.

15. Kalogeris T, Baines CP, Krenz M, Korthuis RJ. Cell biology of ischemia/reperfusion injury. Int Rev Cell Mol Biol 2012; 298: 229-317.

16. Kang PT, Chen CL, Lin P, Zhang L, Zweier JL, Chen YR. Mitochondrial complex I in the post-ischemic heart: reperfusion-mediated oxidative injury and protein cysteine sulfonation. J Mol Cell Cardiol 2018; 121: 190-204.

17. Lamperti A, Conger AD, Jenkins O, Cohen G, Rizzo A, Davis ME, Sodicoff M. WR-2721 entry into the brain across a modified blood-brain barrier. Radiat Res 1988; 115: 303-313.

18. Lee HJ, Kim JS, Song MS, Seo HS, Yang M, Kim JC, Jo SK, Shin T, Moon C, Kim SH. Amifostine ameliorates recognition memory defect in acute radiation syndrome caused by relatively lowdose of gamma radiation. J Vet Sci 2010; 11: 81-83.

19. Ma Z, Xin Z, Di W, Yan X, Li X, Reiter RJ, Yang Y. Melatonin and mitochondrial function during ischemia/reperfusion injury. Cell Mol Life Sci 2017; 74: 3989-3998.

20. Merter AA, Mayir B, Erdogan O, Colak T. Protective effects of amifostine on ischemia-reperfusion injury of rat kidneys. Indian J Pharmacol 2015; 47: 185-189.

21. Mukherjee N, Carroll BL, Spees JL, Delay ER. Pre-treatment with amifostine protects against cyclophosphamide-induced disruption of taste in mice. PLoS One 2013; 8: e61607.

22. Nagy Z, Nardai S. Cerebral ischemia/repefusion injury: From bench space to bedside. Brain Res Bull 2017; 134: 30-37.

23. Nishimura M, Izumiya Y, Higuchi A, Shibata R, Qiu J, Kudo C, Shin HK, Moskowitz MA, Ouchi N. Adiponectin prevents cerebral ischemic injury through endothelial nitric oxide synthase dependent mechanisms. Circulation 2008; 117: 216-223.

24. Nunnari J, Suomalainen A. Mitochondria: in sickness and in health. Cell 2012; 148: 1145-1159.

25. Ozkul A, Akyol A, Yenisey C, Arpaci E, Kiylioglu N, Tataroglu C. Oxidative stress in acute ischemic stroke. J Clin Neurosci 2007; 14: 1062-1066.

26. Pan Y, Wang N, Xia P, Wang E, Guo Q, Ye Z. Inhibition of Rac1 ameliorates neuronal oxidative stress damage via reducing $\mathrm{BCl}$ 2/Rac1 complex formation in mitochondria through PI3K/Akt/ mTOR pathway. Exp Neurol 2018; 300: 149-166.

27. Popović J, Klajn A, Paunesku T, Ma Q, Chen S, Lai B, Stevanović M, Woloschak GE. Neuroprotective role of selected antioxidant agents in preventing cisplatin-induced damage of human neurons in vitro. Cell Mol Neurobiol 2019; 39: 619-636.

28. Rasey JS. Amifostine. Cancer Biother Radiopharm 1999; 14: 331-335.

29. Ryu DS, Kim SH, Kwon JH, Lee DS. Orostachys japonicus induces apoptosis and cell cycle arrest through the mitochondria-dependent apoptotic pathway in AGS human gastric cancer cells. Int J Oncol 2014; 45: 459-469. 
30. Santini V, Giles FJ. The potential of amifostine: from cytoprotectant to therapeutic agent. Haematologica 1999; 84: 1035 1042.

31. Segreto HR, Oshima CT, Franco MF, Silva MR, Egami MI, Teixeira VP, Segreto RA. Phosphorylation and cytoplasmic localization of MAPK p38 during apoptosis signaling in bone marrow granulocytes of mice irradiated in vivo and the role of amifostine in reducing these effects. Acta Histochem 2011; 113: 300-307.

32. Simerabet M, Robin E, Aristi I, Adamczyk S, Tavernier B, Vallet B, Bordet R, Lebuffe G. Preconditioning by an in situ administration of hydrogen peroxide: involvement of reactive oxygen species and mitochondrial ATP-dependent potassium channel in a cerebral ischemia-reperfusion model. Brain Res 2008; 1240: 177-184.

33. Singh A, Kukreti R, Saso L, Kukreti S. Oxidative stress: a key modulator in neurodegenerative diseases. Molecules 2019; 24 1583.

34. Sun L, Zhuang W, Xu X, Yang J, Teng J, Zhang F. The effect of injection of EGb 761 into the lateral ventricle on hippocampal cell apoptosis and stem cell stimulation in situ of the ischemic/ reperfusion rat model. Neurosci Lett 2013; 555: 123-128.

35. Verstappen CC, Postma TJ, Geldof AA, Heimans JJ. Amifostine protects against chemotherapy-induced neurotoxicity: an in vitro investigation. Anticancer Res 2004; 24: 2337-2341.

36. Wang J, Wang A, He H, She X, He Y, Li S, Liu L, Luo T, Huang N, Luo H, Zou K. Trametenolic acid B protects against cerebral ischemia and reperfusion injury through modulation of microRNA-10a and PI3K/Akt/mTOR signaling pathways. Biomed Pharmacother 2019; 112: 108692.

37. Wang Q, Sun AY, Simonyi A, Kalogeris TJ, Miller DK, Sun GY, Korthuis RJ. Ethanol preconditioning protects against ischemia/ reperfusion-induced brain damage: role of NADPH oxidase-derived ROS. Free Radic Biol Med 2007; 43: 1048-1060.

38. Wei J, Wu X, Luo P, Yue K, Yu Y, Pu J, Zhang L, Dai S, Han D, Fei Z. Homerla attenuates endoplasmic reticulum stress-induced mitochondrial stress after ischemic reperfusion injury by inhibiting the PERK pathway. Front Cell Neurosci 2019; 13: 101

39. Wu SZ, Tao LY, Wang JN, Xu ZQ, Wang J, Xue YJ, Huang KY, Lin JF Li L, Ji KT. Amifostine pretreatment attenuates myocardial ischemia/reperfusion injury by inhibiting apoptosis and oxidative stress. Oxid Med Cell Longev 2017; 2017: 4130824.

40. Yalcin S, Kilickap S, Temucin CM, Erman M. Recombinant human erythropoietin in comparison to amifostine against cisplatin-induced peripheral sensorial neurotoxicity in rats. J Exp Clin Cancer Res 2006; 25: 523-527.

41. Yang Z, Weian C, Susu H, Hanmin W. Protective effects of man giferin on cerebral ischemia-reperfusion injury and its mechanisms. Eur J Pharmacol 2016; 771: 145-151.

42. Youle RJ, Strasser A. The BCL-2 protein family: opposing activities that mediate cell death. Nat Rev Mol Cell Biol 2008; 9 : 47-59.

43. Yu Q, Wang B, Zhao T, Zhang X, Tao L, Shi J, Sun X, Ding Q. NaHS protects against the impairments induced by oxygen-glucose deprivation in different ages of primary hippocampal neurons. Front Cell Neurosci 2017; 11: 67.

44. Yu S, Wang C, Cheng Q, Xu H, Zhang S, Li L, Zhang Q, Gu X, Ding F. An active component of Achyranthes bidentata polypeptides provides neuroprotection through inhibition of mito- chondrial-dependent apoptotic pathway in cultured neurons and in animal models of cerebral ischemia. PLoS One 2014; 9 : e109923.

45. Zorova LD, Popkov VA, Plotnikov EY, Silachev DN, Pevzner IB, Jankauskas SS, Babenko VA, Zorov SD, Balakireva AV, Juhaszova M, Sollott SJ, Zorov DB. Mitochondrial membrane potential. Anal Biochem 2018; 552: 50-59. 